\title{
Epidemiologic Features and Clinical Course of COVID-19: a Retrospective Analysis of 19 Patients in Germany
}

\author{
Sara Elgamasy ${ }^{1}$, Eman Elsayed Sakr ${ }^{1}$, Mohamed Gomaa Kamel ${ }^{2}$, Sherief Ghozy ${ }^{3}$, Ghadeer \\ Gamal Awad ${ }^{1}$, Mostafa Mahmoud Fahmy ${ }^{1}$, Ahmed Ghazy ${ }^{1}$, Mohamed Maged Nagaty ${ }^{1}$, \\ and Mostafa Ebraheem Morra ${ }^{1}$ \\ ${ }^{1}$ Affiliation not available \\ ${ }^{2}$ Minia University Faculty of Medicine \\ ${ }^{3}$ Mansoura University Faculty of Medicine
}

June 15, 2020

\begin{abstract}
Background Severe acute respiratory syndrome coronavirus 2 (SARS-CoV-2) causes coronavirus disease-19 (CoVID-19). The virus has exhibited unprecedented human to human transmission and progressed into a pandemic over a short duration. Aim To report the epidemiology; clinical, radiological, and laboratory characteristics; and outcome of SARS-CoV-2 infection in 19 patients in Germany. Methods Retrospective analysis of 19 patients contracting SARS-CoV-2 infection confirmed by PCR in Germany between March 1, 2020 and March 31, 2020. Results In 19 PCR-confirmed SARS-Cov-2 virus infection (median age 69 years; 12 (63\%) males), the most common presentations were Fever (79\%), cough (79\%), fatigue (79\%), and dyspnea (42\%). The most common comorbidities were hypertension (47\%), hypothyroidism (32\%), and cardiac diseases (32\%). According to chest X-ray, $15(79 \%)$ of patients had abnormal chest X-rays; of these, 13 had bilateral chest infiltrates and two had unilateral infiltrates. Lactate dehydrogenase and C-reactive protein (CRP) were elevated in all patients at baseline investigations; $343 \mathrm{U} / \mathrm{L}$ (169-931 U/L) and $8 \mathrm{mg} / \mathrm{dL}(1-17 \mathrm{mg} / \mathrm{dL})$, respectively. Of the 19 cases, 13 (68.4\%) recovered and discharged, 9 (47.3\%) needed ICU admission, and 4 (21.1\%) cases died. Conclusion Among the 19 patients diagnosed with SARS-CoV-2, mild respiratory tract infections symptoms were frequently reported. Some patients required supplemental oxygen and had variable clinical outcomes following supportive and antibiotic treatments. These findings may contribute to the development of more effective strategies of infection control.
\end{abstract}

Epidemiologic Features and Clinical Course of COVID-19: a Retrospective Analysis of 19 Patients in Germany

Short title: "A retrospective analysis of 19 COVID-19 patients"

Sara Elgamasy ${ }^{1}$, Eman Elsayed Sakr ${ }^{2}$, Mohamed Gomaa Kamel ${ }^{3}$, Sherief Ghozy ${ }^{4}$, Ghadeer Gamal Elsayed ${ }^{5}$, Mostafa Mahmoud Fahmy ${ }^{6}$, Ahmed Ghazy ${ }^{7}$, Mohamed Maged Nagaty ${ }^{5}$, Mostafa Ebraheem Morra ${ }^{8, *}$

${ }^{1}$ Department of Internal Medicine, Klinikum Altmühlfranken Weißenburg, Bayern, 91781, Germany

${ }^{2}$ Faculty of Medicine, Menoufia University, Menoufia, Egypt

${ }^{3}$ Faculty of Medicine, Minia University, Minia, 61519, Egypt

${ }^{4}$ Faculty of Medicine, Mansoura University, Mansoura, 35516, Egypt

${ }^{5}$ Faculty of Medicine, Tanta University, Tanta 31524, Egypt 
${ }^{5}$ Faculty of Medicine, Benha University, 13741, Egypt

${ }^{6}$ Faculty of Medicine, Tanta University, Tanta 31524, Egypt

${ }^{7}$ Internal Medicine Department, Hamad General Hospital, Doha, Qatar

${ }^{8}$ Faculty of Medicine, AlAzhar University, Cairo, 11884, Egypt.

Emails:

SE: sara.elgamasy@klinikum-altmuehlfranken.de

EES:emansakr94@gmail.com

MGK: mohamedgomaa@s-mu.edu.eg (ORCID:0000-0001-9848-2965)

SG: sherief_ghozy@yahoo.com (ORCID:0000-0001-5629-3023)

GGE: ghadeergamal1671994@gmail.com

MMF: mostafamahmoud8668421@gmail.com

AG:dr.ahmedazmyghazy@gmail.com

MMN: mohamed13558@fmed.bu.edu.eg

MEM: mostafamorra.stu.6@azhar.edu.eg (ORCID:0000-0002-4910-3321)

*Corresponding author: Mostafa Ebraheem Morra, Faculty of Medicine, AlAzhar University, Cairo, 11884, Egypt (E-mail: mostafamorra.stu.6@azhar.edu.eg, Phone number: +201028432117).

\section{Abstract \\ Background}

Severe acute respiratory syndrome coronavirus 2 (SARS-CoV-2) causes coronavirus disease-19 (CoVID-19). The virus has exhibited unprecedented human to human transmission and progressed into a pandemic over a short duration.

\section{$\operatorname{Aim}$}

To report the epidemiology; clinical, radiological, and laboratory characteristics; and outcome of SARS-CoV2 infection in 19 patients in Germany.

\section{Methods}

Retrospective analysis of 19 patients contracting SARS-CoV-2 infection confirmed by PCR in Germany between March 1, 2020 and March 31, 2020.

\section{Results}

In 19 PCR-confirmed SARS-Cov-2 virus infection (median age 69 years; 12 (63\%) males), the most common presentations were Fever (79\%), cough (79\%), fatigue (79\%), and dyspnea (42\%). The most common comorbidities were hypertension (47\%), hypothyroidism (32\%), and cardiac diseases (32\%). According to chest X-ray, $15(79 \%)$ of patients had abnormal chest X-rays; of these, 13 had bilateral chest infiltrates and two had unilateral infiltrates. Lactate dehydrogenase and C-reactive protein (CRP) were elevated in all patients at baseline investigations; $343 \mathrm{U} / \mathrm{L}(169-931 \mathrm{U} / \mathrm{L})$ and $8 \mathrm{mg} / \mathrm{dL}(1-17 \mathrm{mg} / \mathrm{dL})$, respectively. Of the 19 cases, $13(68.4 \%)$ recovered and discharged, 9 (47.3\%) needed ICU admission, and 4 (21.1\%) cases died.

\section{Conclusion}

Among the 19 patients diagnosed with SARS-CoV-2, mild respiratory tract infections symptoms were frequently reported. Some patients required supplemental oxygen and had variable clinical outcomes following 
supportive and antibiotic treatments. These findings may contribute to the development of more effective strategies of infection control.

\section{Keywords}

Coronavirus - Infection - COVID-19 - Outbreak - Pandemic.

\section{Introduction}

The recent severe acute respiratory syndrome coronavirus 2 (SARS-CoV-2) outbreak is the third novel CoV within the last 17 years. Most patients present with mild symptoms such as fever, cough, expectoration, headache, myalgia or fatigue, or dyspnea $(1,2)$. Some patients report gastrointestinal manifestations that precede respiratory illness and fever by 1-2 days. However, other patients may develop lethal complications such as pneumonia, acute respiratory distress syndrome (ARDS), RNAaemia, and multi-organ failure. It is apparent that SARS-CoV-2 is now much more hazardous than expected compared to previous outbreaks such as the H1N1 pandemic which caused 12,429 deaths over a year while SARS-CoV-2 caused more than 13,000 over 5 weeks in the USA(3). Moreover, the influenza pandemic which infected one third of world's population and resulted in 50 million deaths in 1918 had a basic reproductive number (BRN) of 1.8 only while SARS-CoV-2 has an estimated BRN (R0) of $>2-3(4,5)$.

Unlike other viral infections such as SARS, SARS-CoV-2 is highly contagious with a relatively long asymptomatic period. Moreover, there is evidence of pre-symptomatic transmission of the virus(6). Given the similarity in viral load between symptomatic and asymptomatic patients(7), controlling SARS-CoV-2 transmission is highly challenging. This retrospective analysis reports the epidemiologic features, clinical characters, radiological and laboratory findings, and outcomes of 19 patients with PCR-confirmed SARS-CoV-2 infection in Germany.

\section{Methods}

Study Design

This is a retrospective analysis of medical records, data from patients with confirmed SARS-CoV-2 in Klinikum Altmühlfranken Weißenburg Hospital, Germany from March 1, 2020 to March 31, 2020 were reviewed. All the methods were performed per the relevant approved guidelines, regulations, and declaration of Helsinki. According to the German Medical Association Professional Code of Conduct in Bavaria, ethical approval was not necessary due to the retrospective nature of our investigation and the complete anonymity of reported data(2020-1096) .

Definition and Data Collection

Patients with laboratory-confirmed SARS-CoV-2 using reverse-transcriptase-polymerase-chain reaction (RTPCR) assay of a specimen collected on a nasopharyngeal swab or throat swab regardless of age and gender of the patient were included. A standardized data extraction form was utilized to collect the data from the medical records. The study mentions no identifying information from the participants and all responses were recorded anonymously. The treating doctor in Klinikum altmühlfranken Weißenburg Hospital provided the medical records to the research group. The extraction form entailed epidemiologic data such as the setting of contact and affected family members. We also reported demographic data such as age, gender, and associated comorbidities. Information on clinical symptoms or signs at presentation and X-ray findings were also reported. Moreover, we collected the laboratory markers such as leukocytes and lymphocytes count, red blood cells (RBCs), and hemoglobin. Biochemical variables including lactate dehydrogenase (LDH), alanine aminotransferase (ALT), aspartate aminotransferase (AST), urea, creatinine, C-reactive protein (CRP), and electrolytes were reported. Troponin, procalcitonin, and creatine kinase were also collected. Patients were categorized as either needed mechanical ventilation or not.

Statistical Analysis

Median and range were used to represent continuous variables while we used frequencies and percentages 
to represent categorical variables. All data were analyzed using $\mathrm{R}$ software version 4.0.0 using the package "Rcmdr", and graphically presented using packages "ggplot2", "ggrepel", and "ggpubr".

\section{Results}

Epidemiologic and Clinical Features

Between March 1, 2020 and March 31, 2020, a total of 19 cases with PCR-confirmed COVID-19 were managed in the hospital. The timeline of analyzed cases is shown in Figure 1. Of the 19 patients, 8 and 11 were assorted into needed mechanical ventilation or not, respectively. The median age of patients was 69 (range 45-90); of them, $12(63 \%)$ were males. Nine patients (47\%) had a history of known household contact. Fever (79\%), cough $(79 \%)$, fatigue $(79 \%)$, and dyspnea $(42 \%)$ were the most common presentations. The median temperature was $39^{\circ} \mathrm{C}(37-40)$. Gastrointestinal manifestations were observed in $32 \%$ of cases. Three patients had loss of taste and/or appetite. The median duration from symptom onset to hospital admission was 10 (228) days. Most of subjects had associated comorbidities (89\%); hypertension (47\%), hypothyroidism (32\%), and cardiac problems $(32 \%)$ were the most common reported comorbidities (Table 1) .

Imaging and Laboratory Findings

Baseline auscultation revealed abnormal chest in $14(74 \%)$ of cases; $12(63 \%)$ had bilateral rales or crepitations while two (11\%) had bilateral wheezes. Radiologically, 15 patients $(79 \%)$ had abnormal chest X-rays: 13 with bilateral chest infiltrates and 2 with unilateral infiltrates.

Median total leucocytes count was equal in patients who required $\mathrm{O}_{2}$ supplementation $(\mathrm{n}=11,58 \%)$ to those who did not require $\mathrm{O}_{2}$. Besides, mdian glomerular filtration rate was $62 \mathrm{~mL} / \mathrm{min} / 1.73 \mathrm{~m}^{2}$. Serum urea, creatinine, AST, ALT, and total bilirubin revealed normal to elevated values; $39 \mathrm{mg} / \mathrm{dL}(12-129 \mathrm{mg} / \mathrm{dL})$ and $1 \mathrm{mg} / \mathrm{dL}(0.6-2.2 \mathrm{mg} / \mathrm{dL}), 24 \mathrm{U} / \mathrm{L}(9-117 \mathrm{U} / \mathrm{L}), 50 \mathrm{U} / \mathrm{L}(16-147 \mathrm{U} / \mathrm{L})$, and $0.5 \mathrm{mg} / \mathrm{dL}(0.2-1.6 \mathrm{mg} / \mathrm{dL})$, respectively. The same pattern was observed for procalcitonin $0.12 \mathrm{ng} / \mathrm{mL}(0.02-0.64 \mathrm{ng} / \mathrm{mL})$ in 15 cases. LDH and CRP were elevated in all patients at baseline investigations; $343 \mathrm{U} / \mathrm{L}$ (169-931 U/L) and $8 \mathrm{mg} / \mathrm{dL}$ (1-17 mg/dL), respectively. Creatine kinase was reported in seven cases; with 1,115 and $561 \mathrm{U} / \mathrm{L}$ in two cases and normal range in remaining five. Troponine was assessed in nine cases at baseline 13.09 (90.72-4.75) $\mathrm{pg} / \mathrm{mL}$. Moreover, ferritine level was evaluated in 7 patients, all of them revealed elevated values with an overall median of 824 (287-3429) ng/mL. Furthermore, the median segmented neutrophils and lymphocytes values for 15 cases were $72 \%$ and $15 \%$, respectively. Median values for sodium, potassium, chloride, and calcium showed slight to no elevation, and they were $133,3.9,96.5$, and $2.2 \mathrm{mmol} / \mathrm{L}$, respectively (Table 2)

Figure 2 displays median and range for three laboratory workups(Appendix 1). The Median (range) for TLC was $6 \times 10^{9} / \mathrm{L}\left(4-10 \times 10^{9} / \mathrm{L}\right), 7 \times 10^{9} / \mathrm{L}\left(4-16 \times 10^{9} / \mathrm{L}\right)$, and $7 \times 10^{9} / \mathrm{L}\left(4-11 \times 10^{9} / \mathrm{L}\right)$ for first, second, and third workups, respectively. CRP reached its highest value on admission workup $8 \mathrm{mg} / \mathrm{dL}(1-17 \mathrm{mg} / \mathrm{dL})$, then it decreased to $5 \mathrm{mg} / \mathrm{dL}$ for both the second and third workups. Whereas LDH was higher in the second $363 \mathrm{U} / \mathrm{L}(151-596 \mathrm{U} / \mathrm{L})$ than in the first $343 \mathrm{U} / \mathrm{L}$ (169-931 U/L) and third $305 \mathrm{U} / \mathrm{L}$ (196-523 U/L) workups. Median hemoglobin concentrations were higher in the first $[13 \mathrm{~g} / \mathrm{dL}(11-17 \mathrm{~g} / \mathrm{dL})]$ and second $[13 \mathrm{~g} / \mathrm{dL}$ (8-15 g/dL)] workups as opposed to the third workup [12 g/dL $(7-14 \mathrm{~g} / \mathrm{dL})]$. The highest median platelet count was recorded in the third workup $\left[346 \times 10^{9} / \mathrm{L}\left(138-681 \times 10^{9} / \mathrm{L}\right)\right]$; whereas it measured $216 \times 10^{9} / \mathrm{L}$ $\left(85-487 \times 10^{9} / \mathrm{L}\right)$ and $317 \times 10^{9} / \mathrm{L}\left(84-585 \times 10^{9} / \mathrm{L}\right)$ in the first and second workups, respectively.

\section{Outcomes}

All patients received symptomatic treatment including normal saline and antipyretics. Of 18 patients who received antibiotics, Ampicillin/sulbactam was prescribed for 50\%. Out of 19 cases, 13 (68.4\%) recovered and discharged, 9 (47.3\%) needed ICU admission, and 4 (21.1\%) cases died.

\section{Discussion}

In this study, we report detailed epidemiologic and clinical features of 19 patients with PCR-confirmed SARS-CoV-2 infection in Germany. The most common symptoms at the onset of COIVD-19 were fever, 
fatigue, and cough. The median duration from symptom onset to hospital presentation was 10 (2-28) days. This duration was longer in patients who required supplemental $\mathrm{O}_{2}$ [10 (5-21 days)] compared to patients who did not require $\mathrm{O}_{2}$ [7 (2-28 days)]. Nine (47\%) patients had a history of known household contact. In contrast to a previous study from Beijing, the ratio of severe cases of the COVID-19 infection was $42 \%$ in our study while they had $17.6 \%$ only(8). Moreover, $42 \%$ of our patients required $\mathrm{O}_{2}$ supplementation, and $21 \%$ died. Although this finding should be interpreted cautiously due to the small sample, a previous study in Italy on 1,591 patients reported $81.7 \%$ patients needed respiratory support where $88 \%$ needed invasive endotracheal intubation and $11 \%$ need noninvasive mechanical ventilation as well as $13 \%$ needed oxygen mask. Besides, they showed an ICU mortality rate of $26 \%$ (9). Other previous studies demonstrated that 26 to $33 \%$ of patients might require intensive care $(1,10,11)$ while 76 to $90 \%$ of patients require supplemental oxygen $(1,11,12)$ and they had 4 to $15 \%$ mortality rate $(1,10,11)$.

Thirty-two percent of our cases presented with coexisting gastrointestinal symptoms (abdominal pain, nausea, vomiting, or diarrhea). Indeed, gastrointestinal symptoms have been reported in $2-10 \%$ of SARS-CoV-2 patients in Wuhan, the origin of the infection. Moreover, they occurred 1-2 days before the development of respiratory illness (dyspnea and fever, for example)(1). Zhang et al. have explained this finding after detecting angiotensin-converting enzyme-II receptors, the gate of SARS-CoV-2 into host cells, throughout ileum and colon(13). Accordingly, SARS-CoV-2 virus can potentially be transmitted through both the respiratory as well as gastrointestinal routes.

It is noteworthy as well that hypertension was the most common comorbidity among our patients (47\%). Despite our small sample size, this finding agrees with a large study on 5,700 patients where hypertension coexisted in $56.6 \%$ of positive cases(14). The latter reported obesity and diabetes as the second and third most common comorbidities with rates of $41.7 \%$ and $33.8 \%$, respectively. Whereas our patients revealed hypothyroidism and cardiac diseases in the second position (6 patients; 32\%); and diabetes and benign prostatic hyperplasia equally in the third position (3 patients; $16 \%$ ). Further analysis is required to provide optimum protection and screening facilities to patients at risk.

In our analysis, among the abnormalities aforestated, most patients showed lymphopenia and elevated AST as well as ALT, whereas all patients revealed high CRP and LDH. This is consistent with a recent study that reported a high-accuracy association between abnormal laboratory findings and being positive for SARS$\mathrm{CoV}-2$ to the extent that they can even predict the PCR results(15).

Moving on to diagnosis, diagnosing SAR-CoV-2 based on PCR testing is challenging due to the high rate of false-negative results(16), hence the importance of radiology. CT is the radiological investigation of choice in SARS-CoV-2(17), but chest radiograph was beneficial in our cases. Of note, chest radiograph is considered insensitive early in the course of illness, and it is a screening tool in hospitals with limited resources; it can show abnormalities late in disease. However, chest x-ray revealed abnormal lung infiltrates in $15(79 \%)$ of patients. This is consistent with Wong and colleagues, who studied chest X-ray findings in 64 patients and reported baseline abnormalities in $51(79.7 \%)$ patients in the form of consolidations and ground glass opacities(18). Thence, the cost-effectiveness of chest radiographs worths further research for determining its discriminatory power. Accordingly, health authorities should repeat the PCR, isolate suspected cases, use CT (due to having a higher accuracy) and test as many as possible of suspected cases otherwise infected cases could undetectably transmit the virus.

Indeed, the infection continues to spread in around 212 countries and territories with the confirmed cases and deaths exceeded the total numbers of infected and died patients with SARS and MERS-CoVs. Yet, the real numbers of cases remain undoubtedly much higher due to limited screening and testing. In contrast, the announced relatively low numbers in low and middle-income countries could be attributed to their strict protocols of fewer PCR tests because of limited resources, whereas the Infectious Diseases Society of America has suggested a prioritization of testing. The priority is given mainly to symptomatic healthcare staff, immunocompromised, elderly, and critically-ill patients with severe respiratory distress with no obvious reason. Besides, according to the "iceberg" theory, some countries (the UK) test severe cases only while others test for mild cases as well (Germany) which resulted in a much lower mortality rate between both countries 
( $14.8 \%$ versus $4.4 \%$, respectively). Moreover, these high numbers of infection may be partially attributed to late lockdown and screening since many of these greatly affected countries have big cities and airports for tourists from all over the world.

\section{Conclusions}

Among the 19 patients diagnosed with SARS-CoV-2 infection in Germany, clinical presentation was frequently mild respiratory tract infections. Yet, some patients required supplemental oxygen and had variable clinical outcomes following treatment with supportive and antibiotic treatment. These findings may contribute to the development of more effective strategies of SARS-CoV-2 control.

\section{Figure Legends}

\section{Figure 1. The timeline and outcomes of the 19 included patients.}

Figure 2. Laboratory workups for the 19 included patients.

Abbreviations; TLC: total leukocyte count; RBC: Red blood cells; LDH: Lactate dehydrogenase. Not all patients had all laboratory values assessed at second and third workup.

\section{Acknowledgments}

None.

\section{Funding}

None.

\section{Conflicts of Interest}

All authors have no conflict of interest.

\section{References}

1. Wang D, Hu B, Hu C, Zhu F, Liu X, Zhang J, et al. Clinical characteristics of 138 hospitalized patients with 2019 novel coronavirus-infected pneumonia in Wuhan, China. JAMA. 2020;323(11):1061-9.

2. Xu X-W, Wu X-X, Jiang X-G, Xu K-J, Ying L-J, Ma C-L, et al. Clinical findings in a group of patients infected with the 2019 novel coronavirus (SARS-Cov-2) outside of Wuhan, China: retrospective case series. BMJ. 2020;368.

3. 2009 H1N1 Pandemic (H1N1pdm09 virus) | Pandemic Influenza (Flu) | CDC. https://www.cdc.gov/flu/pandemic-resources/2009-h1n1-pandemic.html (accessed April 9, 2020).

4. Syal K. COVID-19: Herd Immunity and Convalescent Plasma Transfer Therapy. J Med Virol. 2020.

5. Liu Y, Gayle AA, Wilder-Smith A, Rocklov J. The reproductive number of COVID-19 is higher compared to SARS coronavirus. J Travel Med. 2020.

6. Hu Z, Song C, Xu C, Jin G, Chen Y, Xu X, et al. Clinical characteristics of 24 asymptomatic infections with COVID-19 screened among close contacts in Nanjing, China. Science China Life Sciences. 2020:1-6.

7. Zou L, Ruan F, Huang M, Liang L, Huang H, Hong Z, et al. SARS-CoV-2 viral load in upper respiratory specimens of infected patients. New Engl J Med. 2020;382(12):1177-9.

8. Tian S, Hu N, Lou J, Chen K, Kang X, Xiang Z, et al. Characteristics of COVID-19 infection in Beijing. J Infect. 2020.

9. Grasselli G, Zangrillo A, Zanella A, Antonelli M, Cabrini L, Castelli A, et al. Baseline characteristics and outcomes of 1591 patients infected with SARS-CoV-2 admitted to ICUs of the Lombardy Region, Italy. JAMA. 2020. 
10. Zhu N, Zhang D, Wang W, Li X, Yang B, Song J, et al. A novel coronavirus from patients with pneumonia in China, 2019. New Engl J Med. 2020.

11. Huang C, Wang Y, Li X, Ren L, Zhao J, Hu Y, et al. Clinical features of patients infected with 2019 novel coronavirus in Wuhan, China. The Lancet. 2020;395(10223):497-506.

12. Chen N, Zhou M, Dong X, Qu J, Gong F, Han Y, et al. Epidemiological and clinical characteristics of 99 cases of 2019 novel coronavirus pneumonia in Wuhan, China: a descriptive study. The Lancet. 2020;395(10223):507-13.

13. Zhang H, Kang Z, Gong H, Xu D, Wang J, Li Z, et al. The digestive system is a potential route of 2019-nCov infection: a bioinformatics analysis based on single-cell transcriptomes. BioRxiv. 2020.

14. Richardson S, Hirsch JS, Narasimhan M, Crawford JM, McGinn T, Davidson KW, et al. Presenting characteristics, comorbidities, and outcomes among 5700 patients hospitalized with COVID-19 in the New York City area. JAMA. 2020.

15. Mardani R, Vasmehjani AA, Zali F, Gholami A, Nasab SDM, Kaghazian H, et al. Laboratory Parameters in Detection of COVID-19 Patients with Positive RT-PCR; a Diagnostic Accuracy Study. Archives of Academic Emergency Medicine. 2020;8(1).

16. Wang W, Xu Y, Gao R, Lu R, Han K, Wu G, et al. Detection of SARS-CoV-2 in different types of clinical specimens. JAMA. 2020.

17. Yang W, Sirajuddin A, Zhang X, Liu G, Teng Z, Zhao S, et al. The role of imaging in 2019 novel coronavirus pneumonia (COVID-19). Eur Radiol. 2020:1-9.

18. Wong HYF, Lam HYS, Fong AH-T, Leung ST, Chin TW-Y, Lo CSY, et al. Frequency and distribution of chest radiographic findings in COVID-19 positive patients. Radiology. 2020:201160.

Table 1. Baseline Characteristics of Patients Infected with SARS-CoV-2.

\begin{tabular}{|c|c|}
\hline & All patients $(\mathrm{N}=19)$ \\
\hline Demographics & Demographics \\
\hline Age, median (range) y & $69(45-90)$ \\
\hline Male sex, No. $(\%)$ & $12(63)$ \\
\hline Any comorbidity, No. (\%) & $17(89)$ \\
\hline History of contact with a SARS-CoV-2 patient & $9(47)$ \\
\hline Positive Family member & $9(47)$ \\
\hline Days from Symptoms Onset to Hospital Presentation, median (range) & $10(2-28)$ \\
\hline Signs and symptoms on presentation, No. (\%) & Signs and symptoms on presentation, No. (\%) \\
\hline Fever & $15(79)$ \\
\hline Cough & $15(79)$ \\
\hline Fatigue & $13(68)$ \\
\hline Dyspnea & $8(42)$ \\
\hline GIT Manifestations $\mathbb{I}$ & $6(32)$ \\
\hline Poor General Condition & $6(32)$ \\
\hline Dehydration & $6(32)$ \\
\hline Rhinorrhea & $5(26)$ \\
\hline Sputum Production & $4(21)$ \\
\hline Sore throat & $3(16)$ \\
\hline Loss of Taste and/or Appetite & $3(16)$ \\
\hline Headache & $2(11)$ \\
\hline Myalgia & $1(5)$ \\
\hline \multicolumn{2}{|l|}{ Comorbidities, No. (\%) } \\
\hline HTN & $9(47)$ \\
\hline
\end{tabular}




\begin{tabular}{|c|c|c|}
\hline & All patients $(\mathrm{N}=19)$ & $\mathrm{D}$ \\
\hline Hypothyroidism & $6(32)$ & 5 \\
\hline Cardiac Conditions ${ }^{¥}$ & $6(32)$ & 4 \\
\hline Type 2 DM & $3(16)$ & 2 \\
\hline $\mathrm{BPH}$ & $3(16)$ & 2 \\
\hline Obesity & $2(11)$ & 0 \\
\hline Other Chronic Conditions & $8(42)$ & 4 \\
\hline Vital signs at presentation, median (range) & Vital signs at presentation, median (range) & \\
\hline Temperature, ${ }^{\circ} \mathrm{C}$ & $39(37-40)$ & \\
\hline Heart rate, /min & $88(63-116)$ & \\
\hline Systolic blood pressure, $\mathrm{mm} \mathrm{Hg}$ & $142(100-175)$ & \\
\hline Pulse oximeter $\mathrm{O}_{2}$ saturation, $\%$ & $96(78-100)$ & \\
\hline
\end{tabular}

HTN: hypertension; DM: diabetes mellitus; $\mathbb{I}^{\mathbb{I}}$ ncludes Abdominal pain, Nausea, Vomiting, and Diarrhea; ${ }^{¥}$ Includes Atrial fibrillation, chronic heart disease/failure, A-V block, and coronary artery disease.

Table 2. Baseline Clinical Findings of Patients Infected with SARS-CoV-2.

\begin{tabular}{lll}
\hline & All patients $(\mathrm{N}=19)$ & Did not require supplemental O2 (n $=$ \\
\hline Baseline Investigations, median (range) & Baseline Investigations, median (range) & Baseline Investigations, median (range \\
TLC, $\times 10^{9} / \mathrm{L}$ & $6(4-10)$ & $6(4-10)$ \\
$\mathrm{RBC}, \times 10^{6} / \mathrm{L}$ & $5(4-5)$ & $5(4-5)$ \\
Hemoglobin, g/dL & $13(11-17)$ & $14(11-17)$ \\
Segmented Neutrophils, $\%$ & $72(57-88)$ & $68(57-88)$ \\
Lymphocytes, $\%$ & $15(2-28)$ & $18(2-28)$ \\
Platelets, $\times 10^{9} / \mathrm{L}$ & $216(85-487)$ & $208(121-415)$ \\
ALT (U/L) & $24(9-117)$ & $19(9-46)$ \\
AST (U/L) & $50(16-147)$ & $30(16-86)$ \\
Total bilirubin (mg/dL) & $0.5(0.2-1.6)$ & $0.5(0.3-1.6)$ \\
GFR (CKD- EPI), mL/min/1.73 m ${ }^{2}$ & $62(28-117)$ & $54(28-103)$ \\
Urea (mg/dL) & $39(12-129)$ & $35(20-129)$ \\
Serum Creatinine (mg/dL) & $1(0.6-2.2)$ & $1.1(0.6-2.2)$ \\
LDH, U/L & $343(169-931)$ & $299(169-675)$ \\
C-reactive protein, mg/dL & $8(1-17)$ & $6(1-17)$ \\
Procalcitonin (ng/mL) & $0.12(0.02-0.64)$ & $0.12(0.02-0.64)$ \\
$\mathrm{K}^{+}$(mmol/L) & $3.9(3.1-5.1)$ & $3.8(3.1-5.1)$ \\
Na (mmol/L) & $133(128-143)$ & $133(128-143)$ \\
Cl- (mmol/L) & $96.5(91-102)$ & $96.5(91-101)$ \\
Ca ${ }^{2+}$ (mmol/L) & $2.2(1.6-2.5)$ & $2.3(1.6-2.5)$ \\
Baseline Chest radiograph, No. (\%) & Baseline Chest radiograph, No. (\%) & Baseline Chest radiograph, No. (\%) \\
Abnormal Chest Radiograph & $15(79)$ & $8(73)$ \\
Bilateral Lung Infiltrates & $12(63)$ & $5(45)$ \\
Unilateral Lung Infiltrates & $2(11)$ & $2(18)$ \\
Baseline Auscultation Findings, No. (\%) & & \\
Abnormal Auscultation & $14(74)$ & $9(82)$ \\
Bilateral Rales/Crepitation & $12(63)$ & $7(64)$ \\
Bilateral Wheezes & $2(11)$ & $2(18)$ \\
\hline & & \\
& &
\end{tabular}

TLC: total leukocyte count; RBC: Red blood cells; GFR: Glomerular filtration rate; LDH: Lactate dehydro- 
genase; AST: aspartate transaminase; ALT: alanine transaminase.

\begin{tabular}{|c|c|c|c|c|c|c|c|c|c|c|c|c|c|c|c|c|c|c|c|c|c|}
\hline \multicolumn{20}{|c|}{ March 2020} & \multirow{2}{*}{\multicolumn{2}{|c|}{ Final Outcome/Date }} \\
\hline : & 13 & 14 & 15 & 16 & 17 & 18 & 19 & 20 & 21 & 22 & 23 & 24 & 25 & 26 & 27 & 28 & 29 & 30 & 31 & & \\
\hline 1 & & H & & & + & & & & & & & & & & & & & & & जै & 9-Apr \\
\hline 2 & & & & & & & & & & & & & + & H & & & & 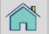 & & & \\
\hline 3 & & & & & & & & $\mathrm{H+}$ & & & & [กี & & 然 & & & & & & & \\
\hline 4 & & & & & & $\mathrm{H}+$ & & & & & & & & & & & & & बैं & & \\
\hline 5 & & & & & & & & & & & & & & & H+ & & & & "m & $\widehat{\Lambda}$ & 23-Apr \\
\hline 6 & & & $\mathrm{H}+$ & & กี & & & & & & & & & & 国理 & & & & & & \\
\hline 7 & & & & & & & & & & & & & & & & & $\mathbf{H}$ & + & [ก] & $+{ }^{110}{ }_{4}$ & 1-Apr \\
\hline 8 & & & & & & & & & & & & & & & $\mathrm{H}+$ & & & & & $\hat{\Omega}$ & 3-Apr \\
\hline 9 & & & & & & & & & & & $\mathrm{H}+$ & & [ก⿵⺆⿻コ一𠃌 & & & & & & & $\begin{array}{c}\text { Ventilation } \\
\text { Weaning }\end{array}$ & 29-Apr \\
\hline 10 & & & & & & & & & + & & & & & Him & & & & & & 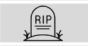 & 12-Apr \\
\hline 11 & H & & & & & & & & & & + & & & & & & & & & $\widehat{\Omega}$ & 6-Apr \\
\hline 12 & & & & & & + & & & & & H & & & & & โักิ & & & & $\widehat{\Omega}$ & 21-Apr \\
\hline 13 & & & & & & $\mathrm{H}+$ & & & & & & & & & & & & & & $\hat{\Lambda}$ & 27-Apr \\
\hline 14 & & & & & & & & & & & & & & & H+ & & & & & $\leftrightarrow$ & 17-Apr \\
\hline 15 & & & & & & & & & & & + & & & & H & & & & & $\widehat{\widehat{\Omega}}$ & 7.Apr \\
\hline 16 & & & & & & & & & & & & & + & & H & & & & & $\widehat{\Omega}$ & 4-Apr \\
\hline 17 & & & & & & & & + & & & & & & H & & & & & & $\hat{\Omega}$ & 8.Apr \\
\hline 18 & & & & & & & & & & & $\mathrm{H}+$ & & & 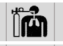 & & & & & & $\hat{\Omega}$ & 29-Apr \\
\hline 19 & & & & & & & $\mathrm{H}+$ & & 11 & & & & & & & & & & & $\widehat{\Lambda}$ & 15-Apr \\
\hline & H & & Imitted & to Hospi & & & & & Reco & verd ar & nd Disch & & & & 国嘼 & & Dece. & & & & \\
\hline & + & & & & & & & \|\|$^{n}$ & & & Req & & & & 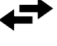 & & & ransferre & & Refused 02 & reatment \\
\hline
\end{tabular}

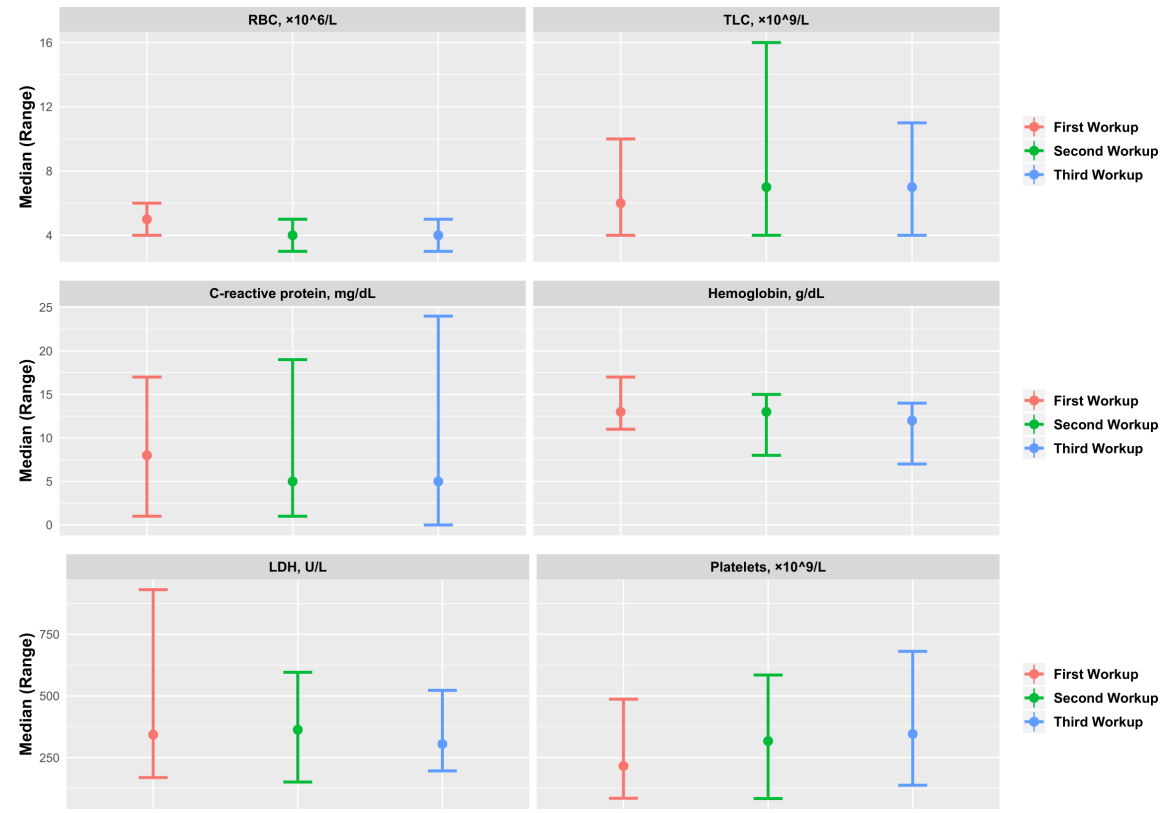

